\title{
A Medical Multimedia based Clinical Decision Support System for Operational Chronic Lung Diseases Diagnosis and Training
}

\author{
Prem Pal Singh Tomar \\ Faculty of Engineering, \\ Dayalbagh Educational \\ Institute, Agra, India
}

\author{
Ranjit Singh \\ Faculty of Engineering, \\ Dayalbagh Educational \\ Institute, Agra, India
}

\author{
P.K Saxena \\ (Rtd.), Faculty of Engineering, \\ Dayalbagh Educational \\ Institute, Agra, India
}

\begin{abstract}
In clinical practice, making decision involves a careful analysis of harms and benefits associated with different treatment options. These decisions, often associated with high stake and important long term consequences, are frequently made in presence of limited resources and information; and an incomplete clinical picture. Under such circumstances, a rigorous and objective analysis of outcomes and probabilities is essential to achieve the best possible decision given a specific clinical situation. This paper presents the use of Case Based Reasoning methodology to develop a Multi Media based Medical Decision Support System for supporting diagnosis of 14 Industrial/operational chronic lung diseases, which consists of 26 symptoms: asbestosis, asthma, bronchitis, byssinosis, emphysema, histoplasmosis, hypersensitivity pneumonitis, influenza, lung cancer, mesothelioma, pneumoconiosis, pneumonia, silicosis and tuberculosis. 173 diagnosed lung disease cases were collected for fourteen major lung diseases, which contains 26 symptoms. After removing the duplicated cases from the database, the system has trained set of 94 cases for lung disease patients. The retrieval strategy using nearest-neighbor approaches is investigated. A Consultant physician's interpretation was used to evaluate the system. The result showed that the system is capable of assisting an inexperience physician in making accurate, consistent and timely diagnoses, also in the study of diagnostic protocol, education, self-assessment, and quality control. In this paper, medical multimedia based clinical decision support system (MMCDSS)is developed for supporting diagnosis of lung diseases from their symptoms and signs through employing Microsoft Visual Basic .NET 2005 along with Microsoft SQL server 2005 environment with the advantage of Object Oriented Programming technology.
\end{abstract}

\section{Keywords}

Medical Multimedia based Clinical Decision Support System, Artificial Intelligence, Case-Based Reasoning, Diagnostic Features, Chronic Lung Diseases, Physician.

\section{INTRODUCTION}

The use of artificial Intelligence (AI) technique i.e. case-based reasoning (CBR), in the development of medical support system has a relatively young history, arose out of the research in cognitive science. The earliest contributions in this area were from Roger Schank and his colleagues at Yale University [1][2]. During the period 1977-1993, CBR research was highly regarded as a plausible high-level model for cognitive processing. It was focused on problems such as how people learn a new skill and how humans generate hypotheses about new situations e cognitive-based researches were to construct decision based on their past experiences. Many prototype of decision support system based on CBR technique were built during this period: for example, Cyrus [3],[4], Mediator [5], Persuader [6], Chef [7], Julia [8], Casey, and Protos [9]. Three CBR workshops were organized in 1988, 1989, and 1991 by the U.S. Defense Advanced Research Projects Agency (DARPA). These formally marked the birth of the discipline of Decision Support System using case-based reasoning.

Computerized evidence-based guidelines and Clinical decision support systems (CDSS) have been promoted as the key to improving effectiveness and efficiency of clinical decisions [14]. Although the use of decision support systems (DSS) in the field of medicine has accelerated in recent years [15].

Many researchers are working on Clinical Decision Support System using CBR with many diverse applications ranging from psychiatry and epidemiology to clinical diagnosi. Most of them aim for a successful implementation of CBR methods to enhance the work of health experts to improve the efficiency and quality of health care. Researchers who have contributed substantially to CBR in medicine include Gierl Schmidt and their colleagues. who focused on a range of applications including children dysmorphic syndromes, antibiotics therapy advising for intensive care and monitoring emerging diseases [20][21]. Notable is their ICONS system [21], first applied to the determination of antibiotic therapy treatment for intensive care then to the prognosis of kidney function defects. For this latter application ICONS learned prototypes associated with graded levels of severity through temporal abstraction [2] and matched new cases with these prototypes to predict the severity of a renal disease [16].

Some real Clinical Support Systems based on CBR technique are: CASEY that gives a diagnosis for the heart disorders [10], GS.52 which is a diagnostic support system for dysmorphic syndromes, NIMON is a renal function monitoring system, COSYL that gives a consultation for a liver transplanted patient [11] and ICONS that presents a suitable calculated antibiotics therapy advise for intensive care patients [12].

Computerized evidence-based guidelines and clinical decision support systems (CDSS) have been promoted as the key to improving effectiveness and efficiency of clinical decisions [17]. Although the use of decision support systems (DSS) in the field of medicine has accelerated in recent years [18].

However, none of the aforementioned studies presented results that showed evidence of first, the inclusion of all the 14 lung diseases perspective; and secondly a system capable 
of assisting a physician who is not specialized in the aspect of lung diseases diagnosis. Thus, this system proposes a multimedia based clinical decision support system for diagnosis of lung diseases as an improvement of earlier works.

\subsection{Decision Support System}

A DSS is an interactive, flexible, and adaptable computer based information system consisting of hardware/software and the human element designed to assist any decision-maker at any level. However, the emphasis is on semi structured and unstructured tasks.

DSS may utilize models, is built by an interactive process (frequently by end-users), supports all the phases of the decision making, and may include a knowledge component.

DSS are designed, built and used to assist in the activities of supporting the decision-making process. And the potential benefits of DSS can be concluded as that improves personal efficiency; expedites problem solving; facilitates interpersonal communication; promotes learning or training; increases organizational control; generates new evidence in support of a decision; creates a competitive advantage over competition; encourages exploration and discovery on the part of the decision maker; reveals new approaches to thinking about the problem space [27].

\section{DSS Components}

\section{Data (Base) Management Subsystem}

2. Model (Base) Management Subsystem

3. Knowledge-based (Management) Subsystem

4. User Interface Subsystem (Dialogue)

5. The User

\section{Architecture of Decision Support System}

Three fundamental components of DSS were identified as, namely: the database management system (DBMS); the model-base management system (MBMS); and the dialogue generation and management system (DGMS) [28]. The DBMS stores information (which can be further subdivided into that derived from an organization's traditional data repositories, external sources such as the Internet or from the personal insights and experiences of individual users). The MBMS handles representations of events, facts, or situations, using various kinds of models. Thirdly, the DGMS, otherwise known as the User interface (UI), is the component that allows users to interact with the system. It was stated that the main product of an interaction with a DSS is insight. As a result, since users of the system are often managers who may not be computer-trained, there is the need to be equipped DSS with intuitive and easy-to use interfaces [29]. The figure (1) gives a precise view of a typical architecture of a DSS.

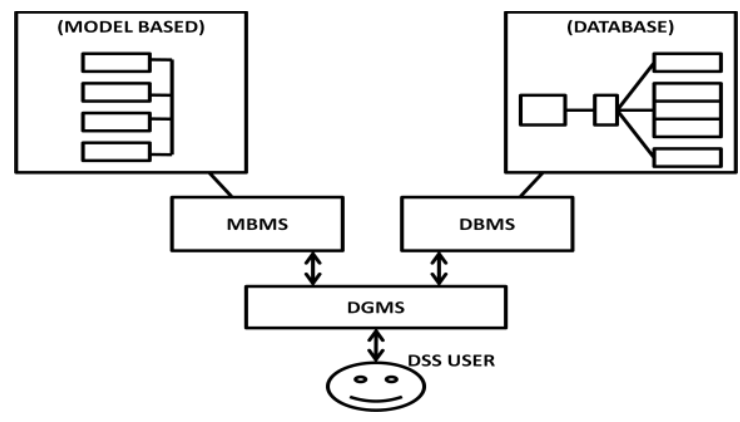

Figure 1: DSS architecture

\subsection{Justification for study}

In clinical practice, making decision involves a careful analysis of harms and benefits associated with different treatment options. These decisions, often associated with high stake and important long term consequences, are frequently made in presence of limited resources and information and an incomplete clinical picture. Under such circumstances, a rigorous and objective analysis of outcomes and probabilities is essential to achieve the best possible decision given a specific clinical situation [24].

Therefore, physician is required to be fully conversant with the diversity of possible patterns, recognize and diagnose them, timely and accurately. Hence, a physician who is not a specialist in the field of the lung diseases has to refer to textbooks and study past diagnosis before concrete diagnosis can be made and conclusion reached. Hence, there is the need for a system, which can assist the physician to reach timely and accurate decision.

\subsection{Knowledge Engineering Tasks In Developing CBR Based Systems}

Case-based Reasoning (CBR) is one problem-solving technology. It processes problems currently encountered by referring to experiences. Depending on experiences accumulated individually, individuals will take different measures in their reasoning principles and thinking procedures against problems. CBR is inspired by the way human's reasoning CBR cycle has four major procedures, as illustrated in Figure1 [25] [26].

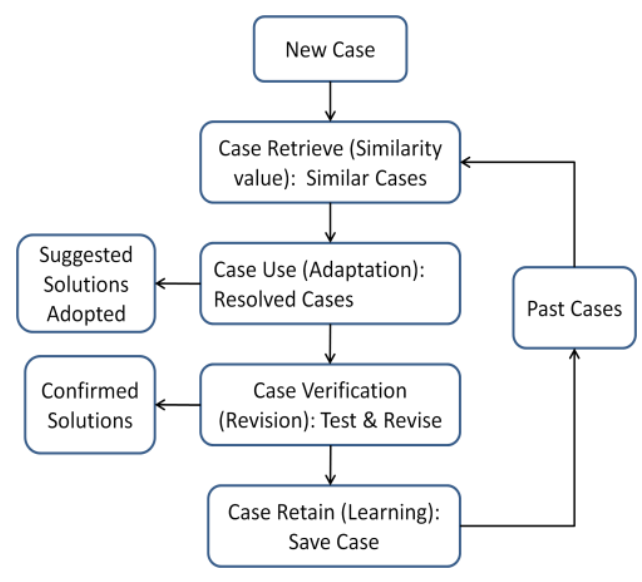

Figure 2: CBR cycle

When a new case comes to the system, it carries out the work of matching. Upon getting exact match, the result of the existing case will be used as the final diagnosis without any change. While in case no exact match is found the nearest neighbor technique looked for whose result is adjusted according to the new case using the adaptation rules and the result is displayed. Such a new case is saved in the case base for future assistance. Accordingly the CBR methodology used, it can be summarized in the following steps:

1. Retrieving: Users input a new case inquiry. The system will search its Case-Memory for an existing case that matches the input problem specification. The indexes are used to retrieve a similar past case from memory. The past case contains the prior solution. 
2. Reusing: The procedure of case reuse is to locate cases that match the new case from the selected cases of the past. If we are lucky (our luck increases as we add new cases to the system), we will find a case that exactly matches the input problem and goes directly to a solution.

3. If we are not lucky, we will retrieve a case that is similar to our input situation but not entirely appropriate to provide as a completed solution. The system must find and modify small portions of the retrieved case that do not meet the input specification i.e. the old solution is modified to conform to the new situation. This process is called "case-adaptation". However, since the probability of complete match between the new and old cases is very small, new cases would be obtained through screening and adaptation according to case differences before a new case is retrieved to users. During adaptation, and after users obtained most similar case, they can browse case contents to acquire the results wanted. Then, save added knowledge and data into database for repetitive use.

4. Revising: From the above step, we can obtain a result to solve problem. Then, send back such a result to conduct case verification to revise and verify the accuracy of the answer. Since the differences between new and old cases might be extremely big or small, professional medical personnel can revise the processing measures themselves. The next time when the same case is encountered, this case will become one of the important cases for reference.

5. Retaining: After a new case is acquired after revision and verification, it can be added to case-base for future use.

A Medical Multimedia based Clinical Decision Support System for diagnosing individual lung disease condition based on the relevant information gathered from the user or patient in the form of the features works in several stages as illustrated in Figure 2.

\section{KNOWLEDGE ACQUISITION}

Knowledge acquisition is a process of acquiring, organizing and studying knowledge for the lung diseases. The data and knowledge of Clinical Decision Support System based on Case-Base Reasoning technique were collected from Biomedical Research Laboratory, Faculty of Engineering, Dayalbagh, Agra, India and Heart, Chest and Allergy Research Laboratory, Agra, India, books and a few electronic websites. This knowledge can be divided by important 26 features, which are shown on table 1.

Table 1: Fact List of Symptoms

\begin{tabular}{|l|l|l|l|}
\hline No. & Chief symptoms & No. & Chief symptoms \\
\hline 1. & Cough & 14 & Alcohol use \\
\hline 2. & Dyspnea & 15 & $\begin{array}{l}\text { Heart rhythm } \\
\text { problem }\end{array}$ \\
\hline 3. & Chest Discomfort & 16 & Abdominal pain \\
\hline 4. & Malaise & 17 & Shoulder pain \\
\hline 5. & Fever & 18 & $\begin{array}{l}\text { difficulty in } \\
\text { swallowing }\end{array}$ \\
\hline
\end{tabular}

\begin{tabular}{|l|l|l|l|}
\hline 6. & Wheezing & 19 & Pain under rib cage \\
\hline 7. & Hemoptysis & 20 & Chemicals exposure \\
\hline 8. & Persistent cough & 21 & Fungi exposure \\
\hline 9. & Fever with chill & 22 & humidifiers exposure \\
\hline 10 & Night sweat & 23 & $\begin{array}{l}\text { Coke oven emissions } \\
\text { exposure }\end{array}$ \\
\hline 11. & Asbestosis exposure & 24 & Silica exposure \\
\hline 12. & Excessive sweating & 25 & Coal dust exposure \\
\hline 13. & Smoking & 26 & Cotton Dust exposure \\
\hline
\end{tabular}

\section{ASSIGNING IMPORTANCE VALUE TO CASE SYMPTOMS}

Features weights for most problem domains are context dependent. The weight assigned to each feature of the case tells how much attention to pay to matches and mismatches in the field when computing the distance measure of a case. Those that are good predictors are then assigned higher importance for matching [10].

The importance of the feature depends upon its prevalence among the diseases. If a feature is common among all diseases like Cough, then it will have the least importance in leading to a diagnosis.

\section{CASE INDEXING AND RETRIEVAL}

Here, we focus our discussion on case indexing and retrieval strategy. Case indexing and retrieval are two separate but closely related processes. Since a case memory may contain thousands of cases, case indices organize their key features with the unique identification number for efficient and reliable case retrieval process for future reuse. Case retrieval searches the case base to find candidate cases that share significant features with the new case. A good review of early literature can be found in [13].

\section{RETRIEVAL USING NEAREST- NEIGHBOR TECHNIQUE}

A common approach for solving classification problems is the use of nearest neighbor methods. Compare each attribute of the new case against that of the old ones in case-base. The case with the highest similarity takes first priority. The system will use the result of the nearest match found and use adaptation process to "revise" this result according to the demands of the novel situation. The system uses the Nearestneighbor algorithm that finds the closest matches of the cases already stored in the database to the new case using a distance calculation, which determines how similar two cases are by comparing their features, the pseudo code of this algorithm [10] can be written as follows:

For each feature in the input case:

Find the corresponding feature in the stored case 
Compare the two values to each other and compute the degree of match

Multiply by a coefficient representing the importance of the feature to the match

Add the results to derive an average match score

This number represents the degree of match of the old case to the input.

A case can be chosen by choosing the item with the largest score.

Nearest-neighbor techniques applied to the retrieval phase of a CBR system (i.e., measuring similarity among cases). Kolodner (1993) propose the algorithm for CBR Similarity, the equation is:

$$
\operatorname{Similarity}(\mathbf{T}, S)=\frac{\sum_{i=1}^{n} \mathbf{f}\left(\mathbf{T}_{\mathbf{i}}, \mathbf{S}_{\mathbf{i}}\right) \times \mathbf{W}_{\mathbf{i}}}{\sum_{\mathbf{i}=1}^{\mathrm{n}} \mathbf{W}_{\mathbf{i}}}
$$

$\begin{array}{ll}\mathrm{T} & \text { Target case } \\ \mathrm{S} & \text { Source case } \\ \mathrm{i} & \text { is an individual attribute } \\ \mathrm{n} & \text { is the number of attributes in each case } \\ \mathrm{Wi} & \text { each individual attribute weight } \\ \mathrm{Ti} & \begin{array}{l}\text { the i number of individual attribute of } \\ \text { input cases }\end{array}\end{array}$

$\mathrm{Si}$

the $\mathrm{i}$ number of individual attribute in case-base

Similarity (T,S) The similarity value of target case and the source case.

represents a typical nearest-neighbor technique that describes a situation for which $\mathrm{T}$ ( Target case) and S ( Source case) are two cases compared for similarity, $\mathrm{n}$ is the number of attributes in each case, $\mathrm{i}$ is an individual attribute from 1 to $\mathrm{n}$, and $\mathrm{Wi}$ is the feature weight of attribute. Similarities are usually normalized to fall within the range 0 to 1 , where 1 means a perfect match and 0 indicates a total mismatch or Similarity Value is less then threshold value.

Consider an illustrative example for implementation of nearest-neighbor technique, Let $\mathrm{T}$ is new case and $\mathrm{C} 1$ and $\mathrm{C} 2$ are old cases

(table 3) then

$$
(\mathrm{T}, \mathrm{C} 1)=74 / 97=0.76289
$$

$$
(\mathrm{T}, \mathrm{C} 2)=69 / 97=0.71134
$$

So, $\mathrm{C} 1$ is the nearest neighbor.

Then the presence of the symptoms in the new and the old case is listed in the next two columns. Local similarity is given in clinical decision support system. The total of all the weights is calculated by adding them which is 97 . Then the sum of weight*similarity is calculated by adding all the products of weight*similarity. In the first comparison the sum is 74 while in the second comparison it is 69 . The sum of weights in the first comparison is: 97

The nearest neighbor value is: $74 / 97=0.76289$

In the second comparison:

The sum of weights is: 69

The sum of weights in the first comparison is: 97

The nearest neighbor value is: $69 / 97=0.71134$

Therefore, case $\mathrm{C} 1$, is the nearest neighbor for the new case $\mathrm{T}$.

\section{MEDICAL MULTIMEDIA SUPPORT}

Multimedia (MM) is an increasingly important tool in training and development for high-technology medical techniques and education [19]. A Medical Multimedia module adds (figure 2) another important dimension i.e. use of Multi Media technologies in Clinical Decision Support System to generate an excellent Multi Media environment by supporting alternate decisions through text, graphics, audio, images, animation and visual impressions and mitigates uncertainty by providing rich information that generate a totally new level of cognitive-style thinking in Clinical Decision Making for more effective and efficient medical decisions [23].

\section{DEVELOPMENT OF THE SYSTEM}

CBR module is designed and developed for supporting diagnosis and management of 14 operational chronic lung diseases: asbestosis, asthma, bronchitis, byssinosis, emphysema, histoplasmosis, hypersensitivity pneumonitis, influenza, lung cancer, mesothelioma, pneumoconiosis, pneumonia, silicosis and tuberculosis chronic lung diseases from their signs and symptoms using Case Based Reasoning methodology that focuses on the reutilization of past experience. It is based on solutions, information and knowledge available in similar problems previously solved. CBR Module is employing Microsoft Visual Basic .NET 2005 along with Microsoft SQL server 2005 environment with the advantage of Object Oriented Programming technology.

The symptoms/facts are aquired from the user by using a specialy designed graphical user interface in Microsoft Visual Basic .NET 2005 as shown in figure 3(1) to figure 3(6) and again in figure 4(1) to 4(6). When a new case comes to the system/ CBR Module, the system carries out the work of matching. The nearest-neighbor approach, retrieve cases based on a weighted sum of features in the input cases that match the cases in case base (database). Every feature/symptom in the input cases is matched to its corresponding feature/symptom in the stored or old cases. Upon getting exact match same result is displayed (shown in Figure 3(7)) as solution.

if no exact match is found the nearest neighbor is looked for all 14 occupational diseases cases available in the database, whose result of the of best case for each proposed disease is graphically displayed as shown in figure 4(7), and the best previous case recovered among the chronic lung diseases is adjusted according to the new case using the adaptation process. New Case indexing involves assigning indexes to cases to facilitate their retrieval. Indexes organize and label cases so that appropriate cases can be done efficiently and 
accurately. Such a new case is also saved in the case base for future assistance.

\section{Adaptation Process}

The main hurdle in diagnosing the chosen lung diseases is the great similarity between their symptoms. Thus, when designing such a diagnostic system this similarity comes up as one of the first challenges. The first and foremost step is to compare the symptoms and segregate the distinguishing ones for the purpose of diagnosis. In order to achieve this, the symptoms for each disease are tabulated and compared. The adaptations process can use the rule based inference and/or medical professional personnel to 'revise' the solution produced using the nearest neighbor technique in producing a result.

\section{Saving New Case}

CBR Module is designed to successfully retain novel problems, however saving a new case has been totally left to the choice of a system user. If he/she chooses to 'save' a new case, after the results have been displayed. Any case saved can be successfully used for future retrieval and reuse by the system.

To generate an excellent medical multimedia environment, multimedia support tools like textual description (Figure 3(10)), video (Figure 3(12)), slide show (Figure 3(14)), website link (Figure 3(16)), pictures and graphs (Figure 3(18)) and Audio (Figure 3(19)), for diagnosed disease. CBR module allows visual simulations, providing rich information and generates a totally new level of cognitive-style thinking in Clinical Decision Making for more effective and efficient medical decisions.

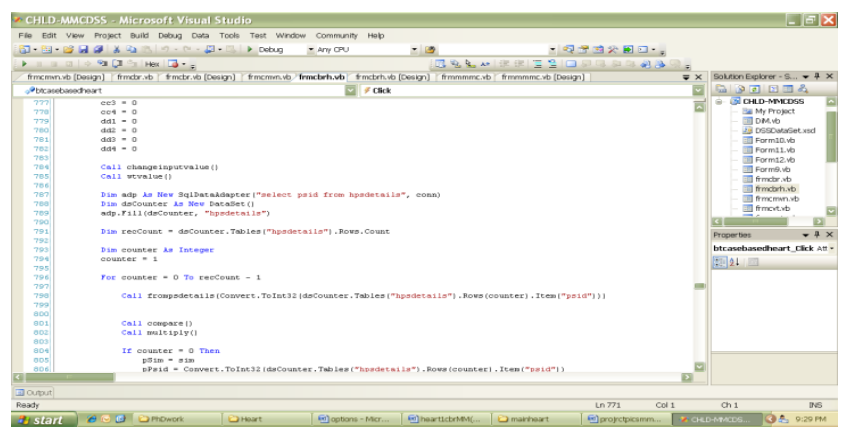

Figure 5: Microsoft Visual Basic .NET 2005, Code window

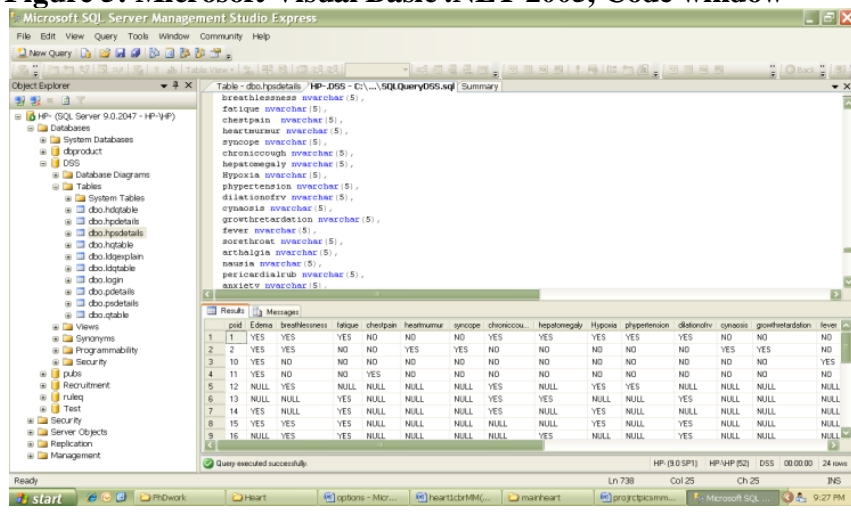

Figure 6: Microsoft SQL server 2005, Database window
To better understand the performance of a diagnostic test, the medical profession defines a number of other measurements. Let us begin by examining a contingency table (Table 2). Contingency tables contain four variables: true positives (TP), true negatives (TN), false positives (FP), and false negatives (FN).

(1) True positive (TP):

The diagnostic system yields positive test result for the sample and thus the sample actually has the disease;

(2) False positive (FP): The diagnostic system yields positive test result for the sample but the sample does not actually have the disease;

(3) True negative $(\mathrm{TN})$ :

The diagnostic system yields negative test result for the sample and the sample does not actually have the disease; and

(4) False negative (FN):

The diagnostic system yields negative test result for the sample but the sample actually has the disease.

\section{RESULTS OF THE CBR MODULE}

To determine the performance of CBR Module a Consultant physician's interpretation was used, an experiment with 273 lung disease patients having background of occupational exposure as mentioned in our study, is performed at Biomedical Research Laboratory, Faculty of Engineering, Dayalbagh Educational Institute (Deemed University), Dayalbagh, Agra, India and Heart, Chest and Allergy Research Laboratory, Agra, India. The testing of CBR Module results in all correct answers except for 6 false positives and 5 false negatives.

The most common measurements in the medical field are sensitivity and specificity, which are defined as:

$$
\begin{aligned}
& \text { Sensitivity }=\frac{\mathbf{T P}}{\mathbf{T P}+\mathbf{F N}} \times \mathbf{1 0 0} \%=97.36 \% \\
& \text { Specificity }=\frac{\mathbf{T N}}{\mathbf{T N}+\mathbf{F P}} \times \mathbf{1 0 0} \%=92.77 \%
\end{aligned}
$$

Two measurements often used in conjunction with sensitivity and specificity are the false-negative rate (FNR) and the falsepositive rate (FPR), also known as the false-alarm rate (FAR):

$$
\begin{aligned}
& \text { FNR }=\frac{\text { FN }}{\text { FN }+ \text { TP }}=1-\text { sensitivity }=0.0264 \\
& \text { FAR }=\frac{\text { FP }}{F P+T N}=1-\text { specificity }=0.0723
\end{aligned}
$$

The false-negative rate and false-alarm rate are probabilities associated with a test inaccurately diagnosing a patient. The false-negative rate represents the probability of a test failing to detect a disorder that is present, whereas, the false-alarm rate represents the probability of a test falsely indicating that a patient has a disorder. Alternates to sensitivity and specificity are the positive predictive value (PPV) and negative predictive value (NPV):

$\mathbf{P P V}=\frac{\mathbf{T P}}{\mathbf{T P}+\mathbf{F P}} \times \mathbf{1 0 0} \%=96.85 \%$ 


$$
\mathbf{N P V}=\frac{\mathbf{T N}}{\mathbf{T N}+\mathbf{F N}} \times \mathbf{1 0 0} \%=93.90 \%
$$

The final mathematical expression commonly used in the medical field is the likelihood ratio (LR). The likelihood ratio is the odds that a specific test result is given to a patient with the disorder compared to the same test result being given to a patient without the disorder. There are two types of likelihood ratios, LR+ and LR-, which can be calculated as follows:

$$
\begin{aligned}
& \mathbf{L R}^{+}=\frac{\text { Sensitivity }}{\text { FAR }}=13.4661 \\
& \mathbf{L R}^{-}=\frac{\text { FNR }}{\text { Specificity }}=0.02846
\end{aligned}
$$

Where $\mathrm{LR}^{+}$is the odds that a positive test result occurs for a patient with the disorder versus one without the disorder and $\mathrm{LR}^{-}$is the odds that a negative test result occurs for a patient with the disorder verses one without the disorder. Thus, good diagnostic tests should have a high $\mathrm{LR}^{+}$and a low $\mathrm{LR}^{-}$. For good CBR Module, diagnostic tests should have a high $\mathrm{LR}^{+}$ and a low $\mathrm{LR}^{-}$as indicated in the results.

\section{SUMMARY AND CONCLUSION}

In this paper we presented a medical multimedia based clinical decision support system, which could be used by stakeholders for arriving at very vital decisions regarding the diagnosis of operational chronic lung diseases. The focus was on the development of a system that can assist physician, especially those who may not be specialist in the area of lung diseases treatment. Thus, the system attempts to improve the effectiveness of diagnosis (in relation to accuracy, timeliness and quality) that is performed by a human physician, rather

Figure 3: Diagnostic and multimedia support windows for exact

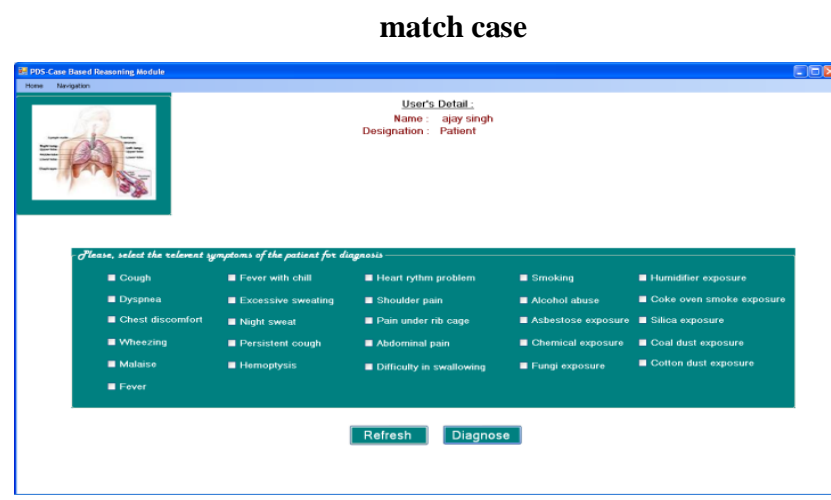

Figure 3(1)

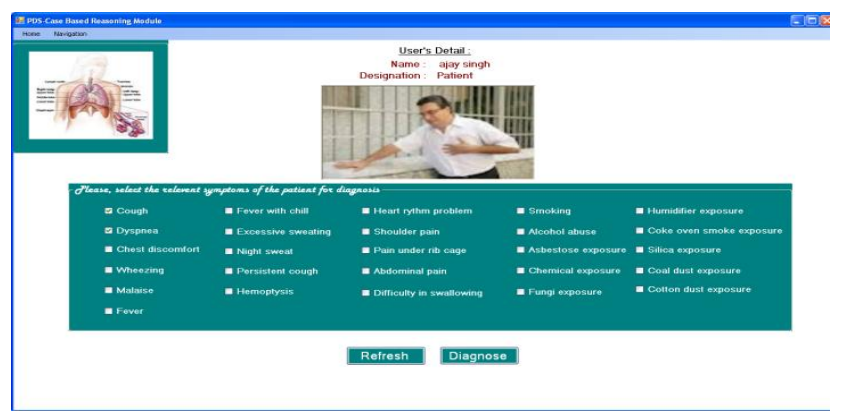

Figure 3(3) than improve their efficiency with respect to decision making. Therefore, the diagnoses made by the system are at least as good as those made by a human expert.

From the development and analysis of medical decision support system, it is evident that CBR technique of Artificial Intelligence (AI) is appropriate methodology for all medical domains and tasks for the following reasons: cognitive adequateness, explicit experience and subjective knowledge, automatic acquisition of subjective knowledge, and system integration.

CBR technique presents an essential technology of building intelligent medical decision support system for medical diagnoses that can aid significantly in improving the decision making of the physicians.

The use of Microsoft Visual Basic .NET 2005 along with Microsoft SQL server 2005 as database is found to be very effective in producing the system under windows environment. For future work, more cases will be added to the case memory.

Future research involves more intensive testing using a larger database from local foundry and diesel engine industries to get more accurate results.

\section{ACKNOWLEDGEMENT}

Authors would like to thank Dr. Varun Kumar Chaudhary, M.B.B.S., M.D., Heart, Chest and Allergy Specialist, Agra, India, for his help in classifying, revising, and evaluating Multi Media based Clinical Decision Support System for operational lung diseases.

The authors also thank the anonymous reviewers for their valuable suggestions and comments.

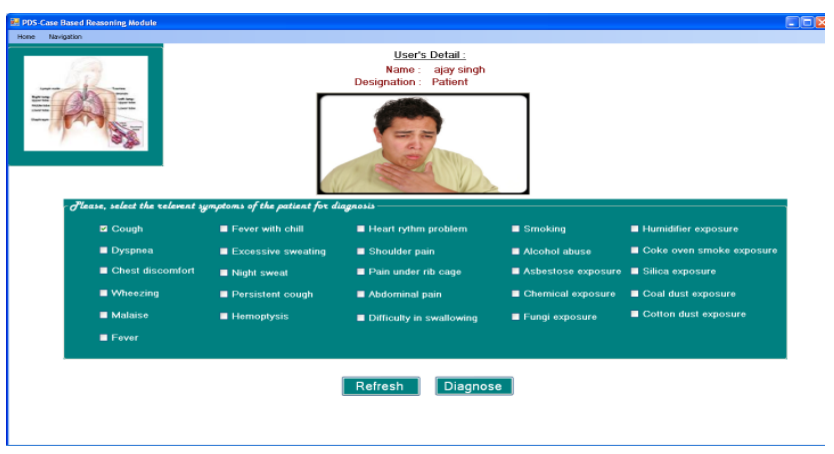

Figure 3(2)

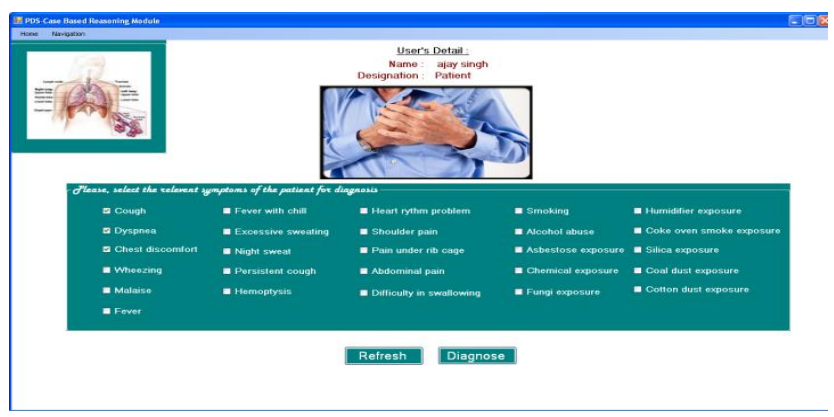

Figure 3(4) 


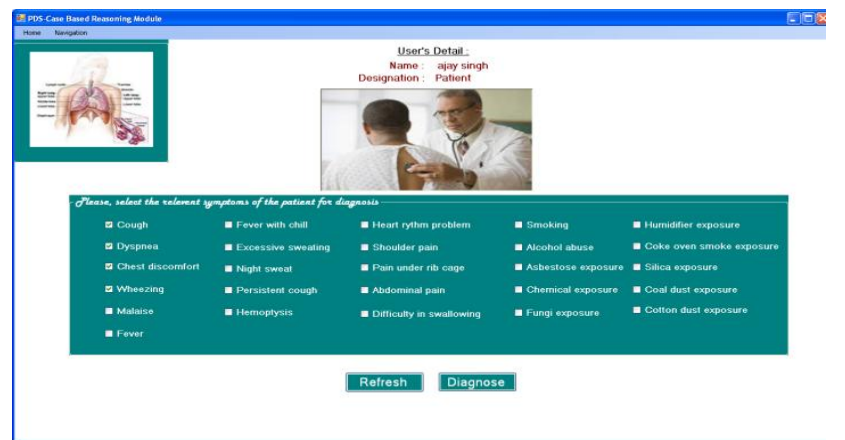

Figure 3(5)

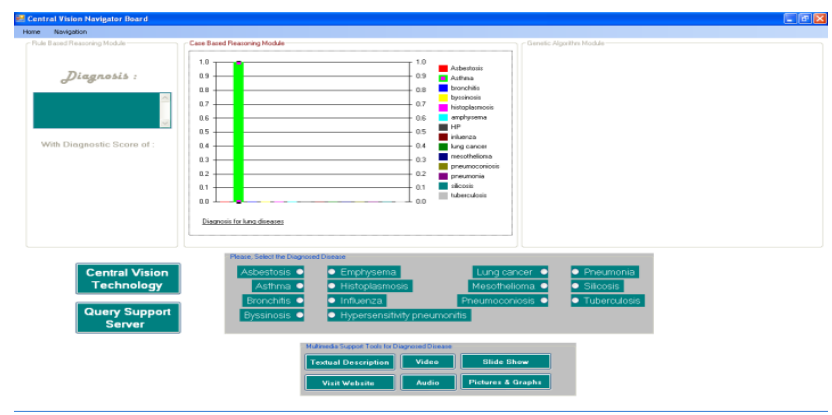

Figure 3(7)

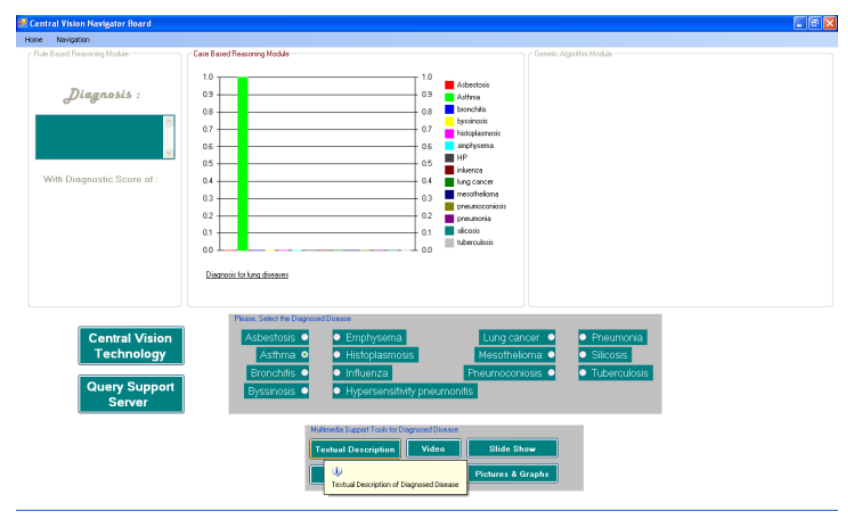

Figure 3(9)

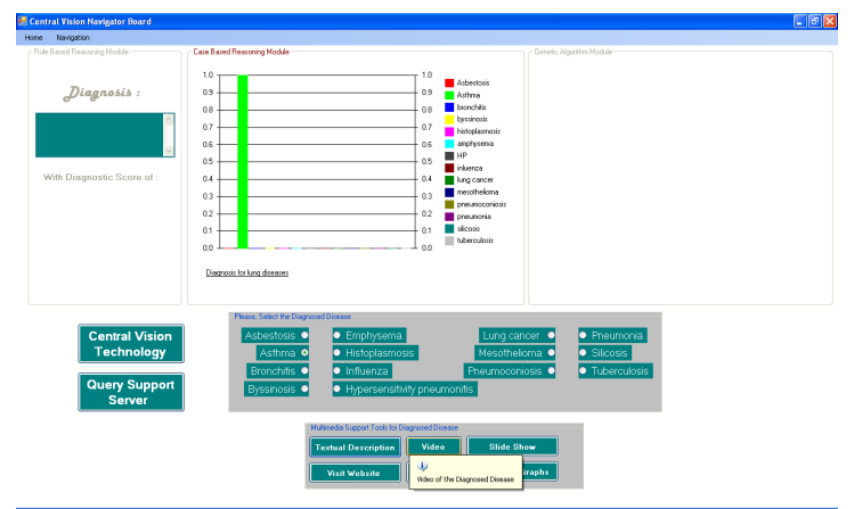

Figure 3(11)

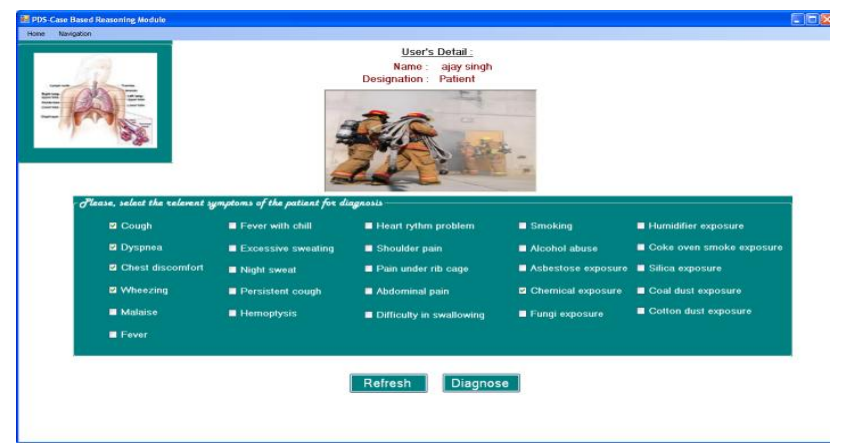

Figure 3(6)

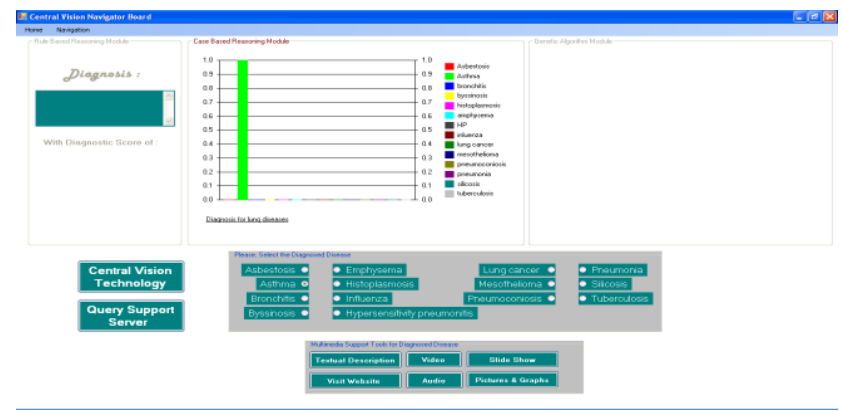

Figure 3(8)

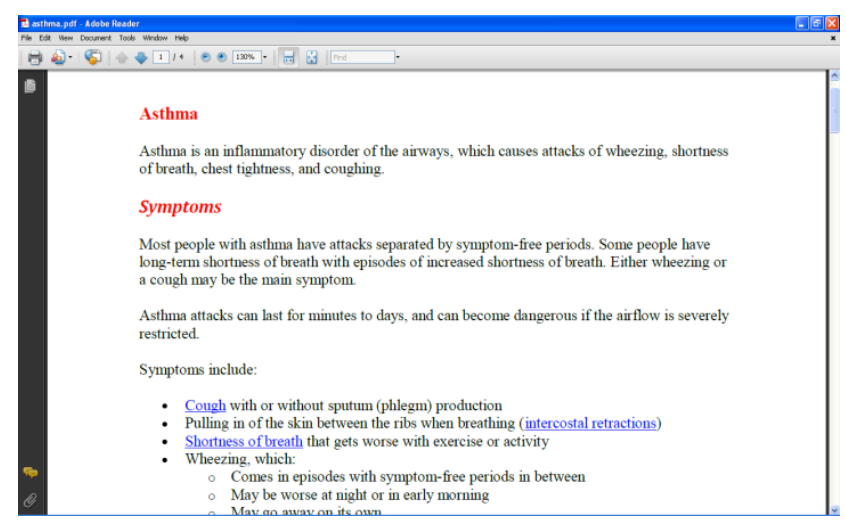

Figure 3(10)

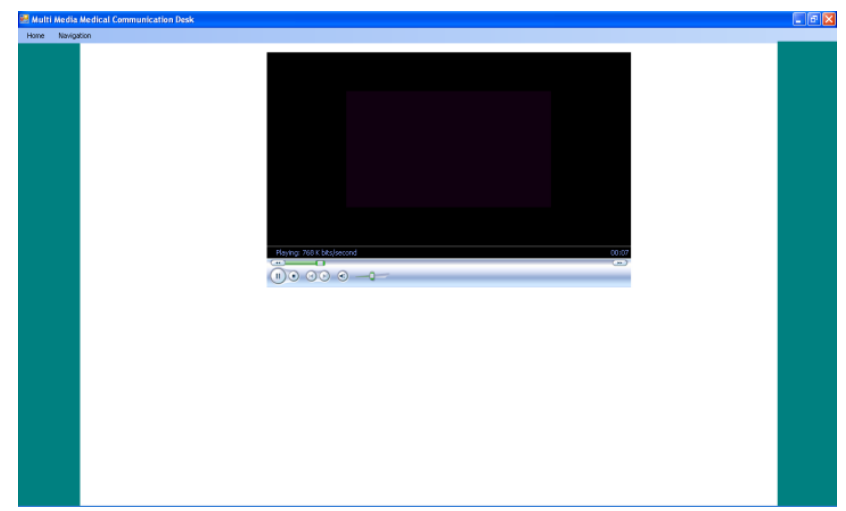

Figure 3(12) 


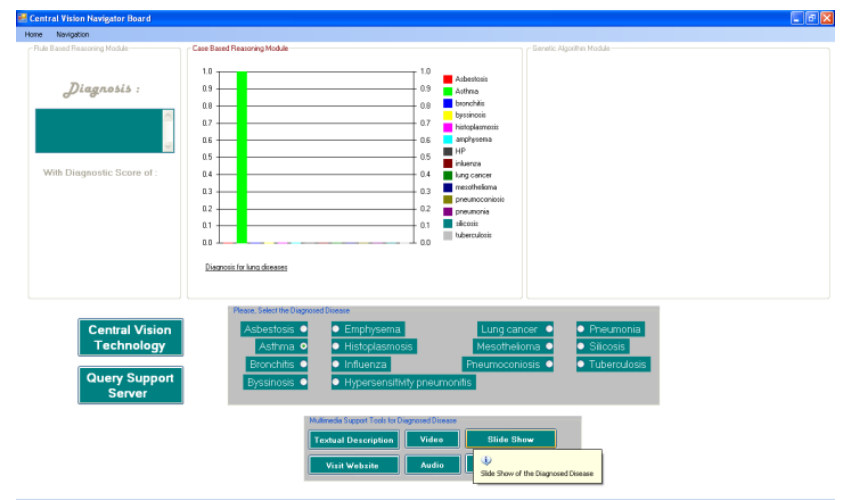

Figure 3(13)

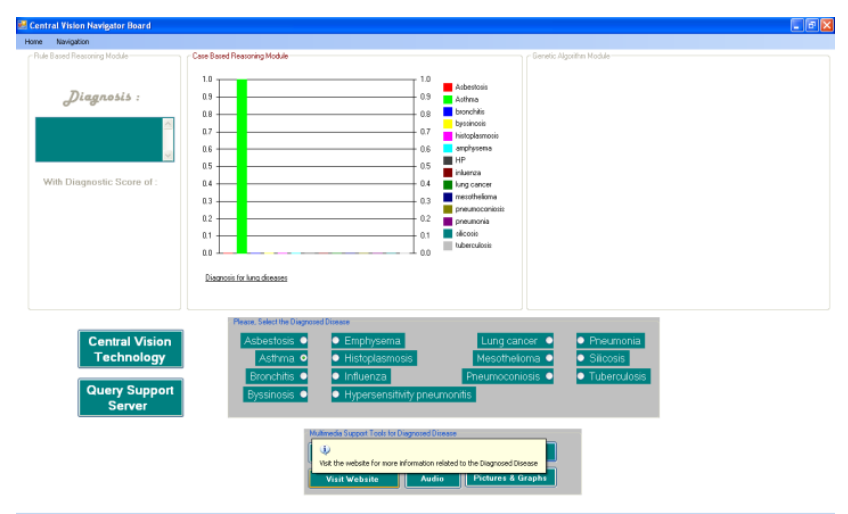

Figure 3(15)

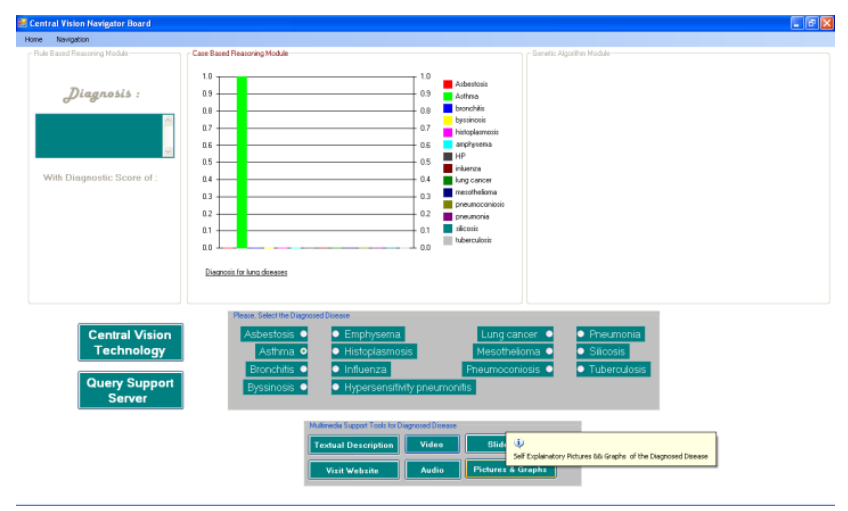

Figure 3(17)

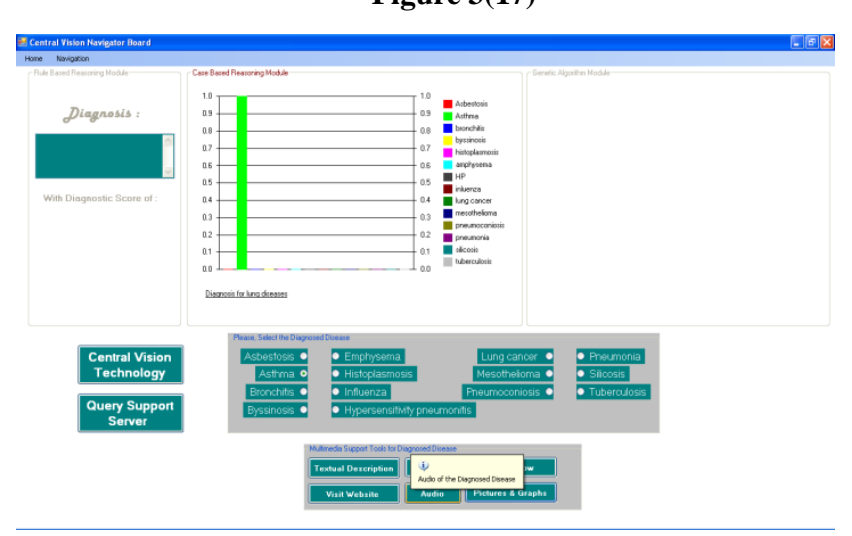

Figure 3(19)

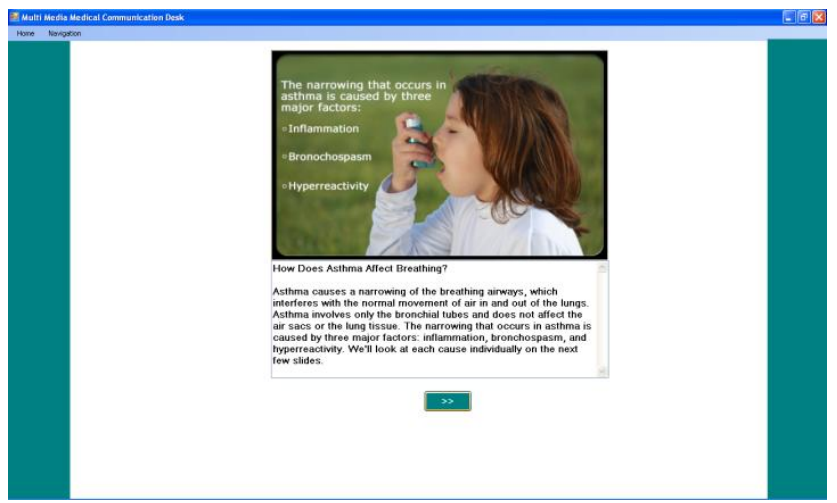

Figure 3(14)

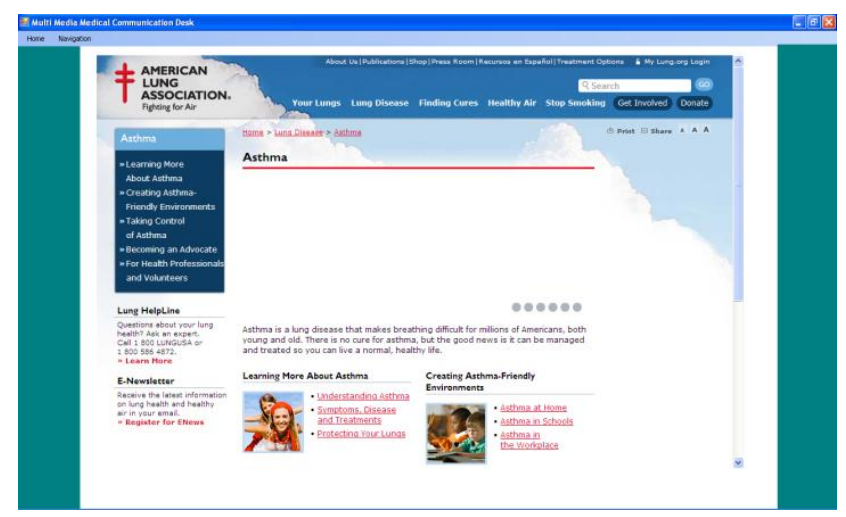

Figure 3(16)

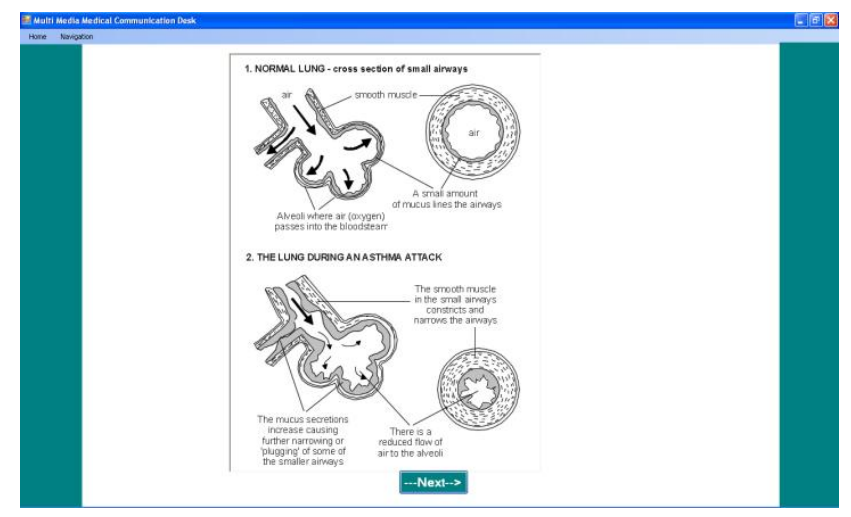

Figure 3(18) 
Figure 4: Diagnostic and multimedia support windows for no exact match case

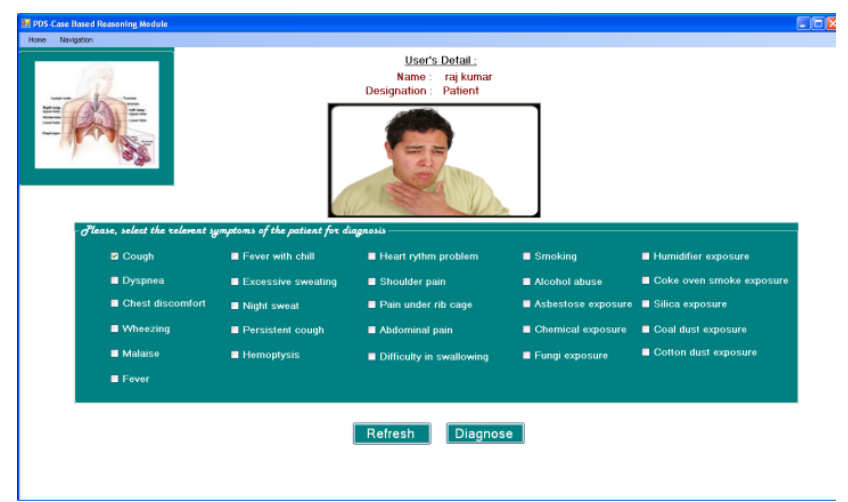

Figure 4(1)

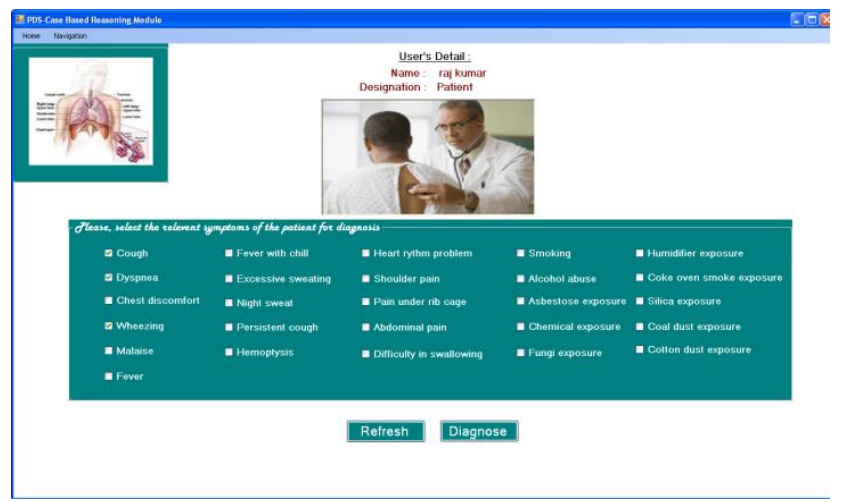

Figure 4(3)

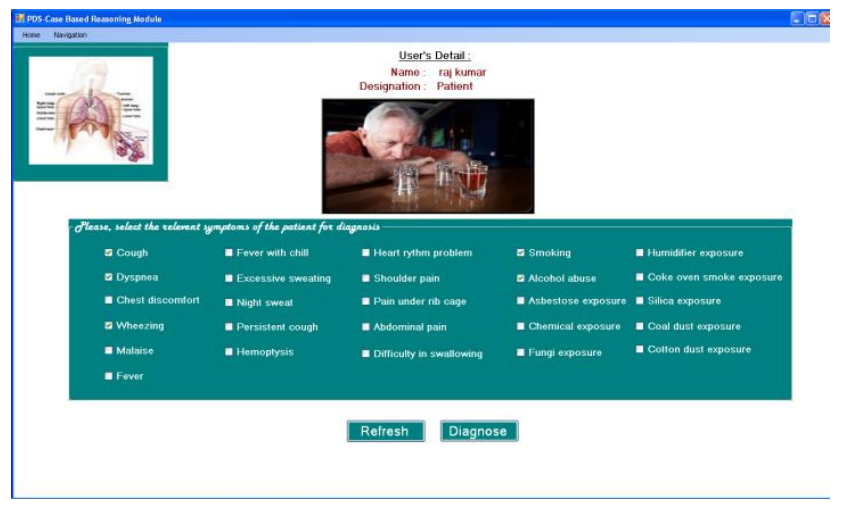

Figure 4(5)

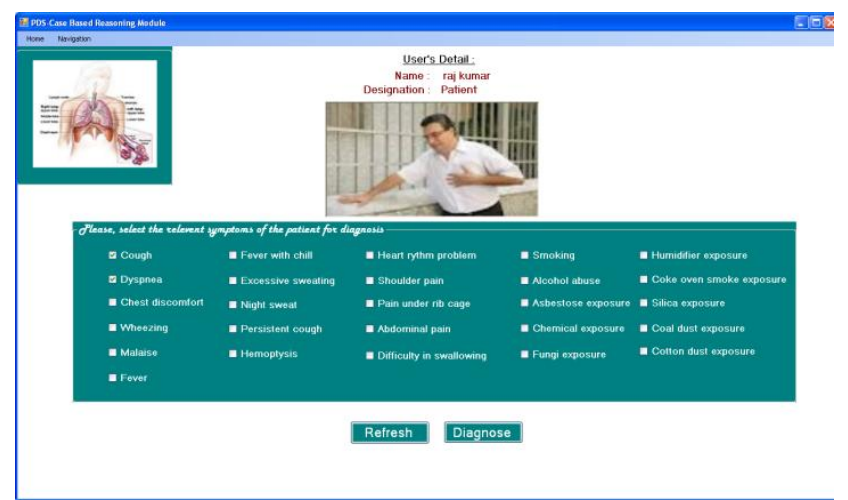

Figure 4(2)

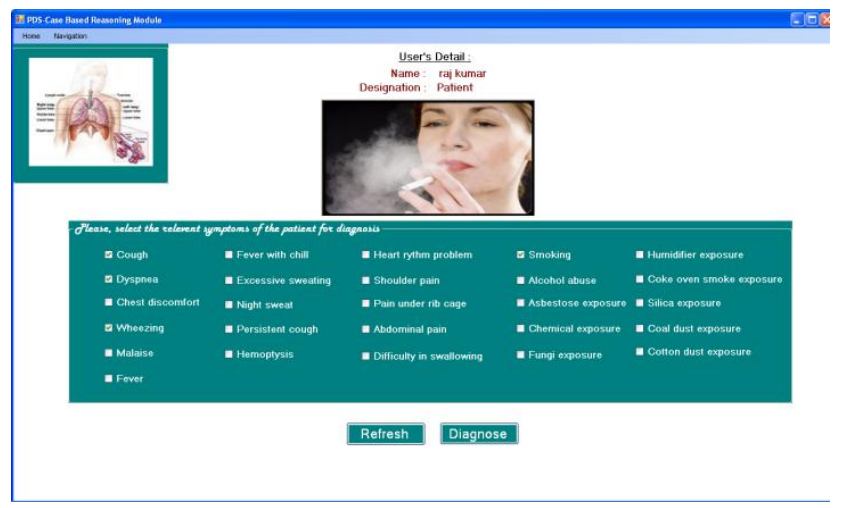

Figure 4(4)

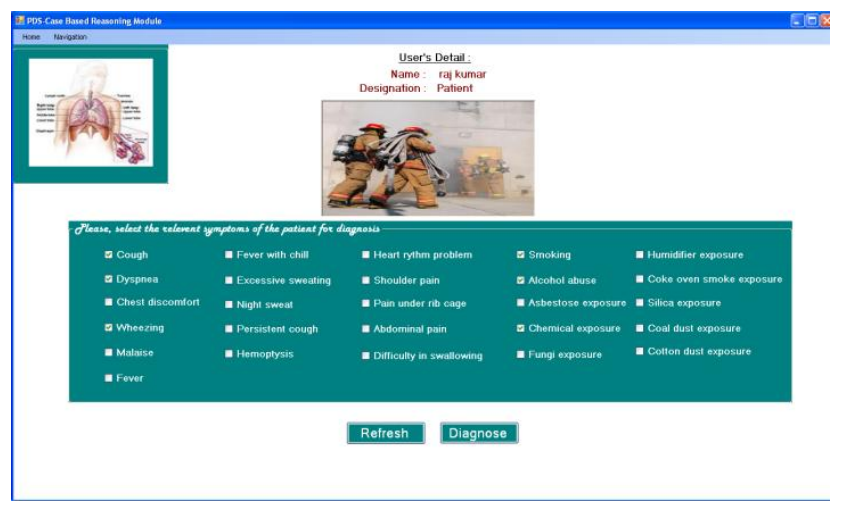

Figure 4(6)

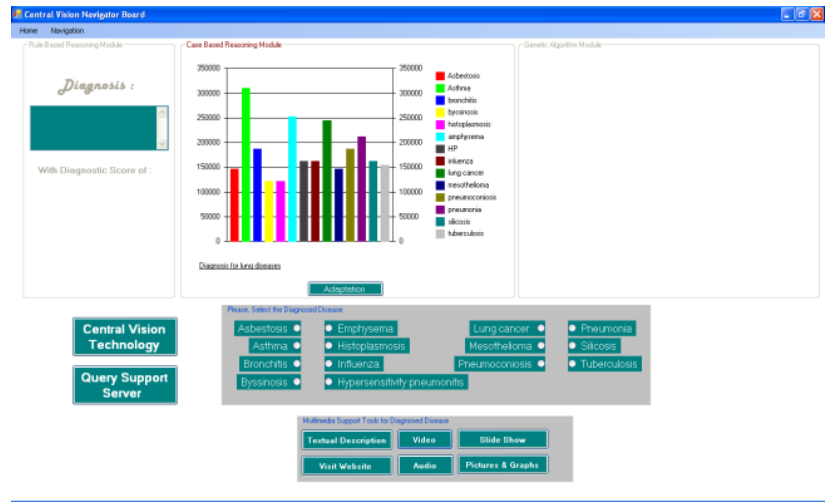

Figure 4(7) 
Table 2: Contingency table

\begin{tabular}{|l|c|c|c|}
\hline Test Results & Disorder present & Disorder absent & Total \\
\hline Positive & TP & FP & TP + FP \\
\hline Negative & FN & TN & \\
\hline Total & TP + FN & FP + TN \\
\hline
\end{tabular}

Table 3: Nearest neighbor calculation

\begin{tabular}{|c|c|c|c|c|c|c|c|c|c|}
\hline S.No. & Symptoms & Weight & $\begin{array}{l}\text { New } \\
\text { Case } \\
T\end{array}$ & $\begin{array}{l}\text { Old } \\
\text { Case } \\
\text { C1 }\end{array}$ & $\begin{array}{l}\text { Loc. } \\
\text { Sim. }\end{array}$ & Wei*Sim & $\begin{array}{l}\text { Old } \\
\text { Case } \\
\text { C2 }\end{array}$ & $\begin{array}{l}\text { Loc. } \\
\text { Sim. }\end{array}$ & Wei*Sim \\
\hline 1. & Cough & 2 & yes & yes & 1 & 2 & yes & 1 & 2 \\
\hline 2. & Dyspnea & 2 & yes & yes & 1 & 2 & no & $\mathbf{0}$ & $\mathbf{0}$ \\
\hline 3. & Malaise & 3 & yes & yes & $\mathbf{1}$ & 3 & yes & 1 & 3 \\
\hline 4. & $\begin{array}{l}\text { Chest } \\
\text { Discomfort }\end{array}$ & 3 & no & Yes & $\mathbf{0}$ & $\overline{\mathbf{0}}$ & Yes & $\mathbf{0}$ & $\overline{\mathbf{0}}$ \\
\hline 5. & Fever & 3 & yes & yes & 1 & 3 & yes & 1 & 3 \\
\hline 6. & Wheezing & 3 & no & yes & $\mathbf{0}$ & $\mathbf{0}$ & yes & $\mathbf{0}$ & $\mathbf{0}$ \\
\hline 26. & Cotton Dust & 5 & yes & yes & 1 & 5 & yes & 1 & 5 \\
\hline & Sum & 97 & & & & 74 & & & 69 \\
\hline
\end{tabular}




\section{REFERENCES}

[1] R. Schank and R. Abelson (eds.), Scripts, Plans, Goals and Understanding, Lawrence Erlbaum Associates, Hillsdale, NJ, 1977

[2] R. Schank, Dynamic Memory: A Theory Of Reminding and Learning in Computers and People, Cambridge University Press, New York, 1982

[3] J. L. Kolodner, Maintaining organization in a dynamic long term memory, Cognitive Science, vol. 7, 243-280, 1983.

[4] J. L. Kolodner, Reconstructive memory, a computer model, Cognitive Science, vol. 7,281-328, 1983.

[5] R. L. Simpson, A computer model of case-based reasoning in problem solving: an investigation in the domain of dispute mediation, Ph.D. dissertation, School of Information and Computer Science, Georgia Institute of Technology, Atlanta, GA, 1985.

[6] K. Sycara, Using case-based reasoning for plan adaptation and repair, in Proceedings of the Case-Based Reasoning Workshop, DARPA, Clearwater Beach, FL, Morgan Kaufmann, San Francisco, 425-434, 1988.

[7] K. J. Hammond, Case-Based Planning, Academic Press, San Diego, CA, 1989.

[8] T. R. Hinrihs, Problem Solving in Open Worlds, Lawrence Erlbaum Associates, Hillsdale, NJ, 1992.

[9] R. Bareiss, Exemplar-Based Knowledge Acquisition: A Unified Approach to Concept Representation, Classification, and Learning, Academic Press, San Diego, CA, 1989.

[10] J. L. Kolodner (Ed.), Case-Based Reasoning, Morgan Kaufmann Publishers: California, 1993

[11] M. Lenz, S. Wess, H. Burkhard and B. Bartsch, CaseBased Reasoning Technology from Foundations to applications, Springer, 1998.

[12] B. Heindl. et al,: A Case-Based Consiliarius for Therapy Recommendation (ICONS): Computer-Based Advice for Calculated Antibiotic Therapy in Intensive Care Medicine. Computer Methods and Programms in Biomedicine 52, 117-127, 1997.

[13] E. L. Rissland, J. Kolodner, \& D. Waltz, Case-based reasoning from DARBA: "Machine learning program plan". Proceedings of the case-Based Reasoning Workshop. San Mateo, CA: Morgan Kaufmann, 113,1998

[14] M Fieschi, J-C Dufour, P Staccini, et al., 2003, "Medical decision support systems: old dilemmas and new paradigms? Tracks for successful integration and implementation,” Methods Inform Med, 42:190-198.8

[15] Vitali Sintchenkoa, Enrico Coierac and Gwendolyn L. Gilberta, 2008 , "Decision support systems for antibiotic prescribing," Current Opinion in Infectious Diseases, $21: 573-579$

[16] Alec Holt, Isabelle Bichindaritz, Rainer Schmidt, And Petra Perner, 2006, "Medical applications in case based reasoning," The Knowledge Enineering review, Vol.20:3, 289-292 , Cambridge University Press
[17] B Graham, AS Detsky, the application of decision analysis to the surgical treatment of early osteoarthritis of wrist. J Bone Joint Surg. Br 2001, 83, 650-4

[18] AS Detsky, G Nalie, MD Krahn, D Naimark, DA Redelmeier. Primer on medical decision analysis: Part 1Getting started. Med Decision Making 1997: 17:123-5

[19] Abdul Hamid M. Ragab, Khalid Abdullah Fakeeh AND Mohamed Ismail Roushdy King ABDULAZIZ, 2004. “A Medical Multimedia Expert System for Heart Diseases Diagnosis \& Training", Proc. $2^{\text {nd }}$ Saudi Sci. Conf., Fac. Sci., KAU, 15-17 March, Part IV, 31-45.

[20] Schmidt, R and Gierl, L, 2001, Temporal abstractions and case-based reasoning formedical course data. Two prognostic applications. In Perner, P (ed.) Proceedings of the Second International Workshop on Machine Learning and Data Mining in Pattern Recognition (Lecture Notes in Computer Science, 2123). Heidelberg: Springer, pp. 23-34

[21] Gierl, L, 1993, ICONS: cognitive basic functions in a case- based consultation system for intensive care. In Andreassen, $\mathrm{S}$ et al. (eds) Proceedings of the Artificial Intelligence in Medicine, AIMED93, pp. 230-236.

[22] P.P.S Tomar, R. Singh, P. K. Saxena \& J. Sharma, Case Based Medical Diagnosis of Occupational Chronic Lung Diseases From Their Symptoms and Signs, International Journal of Biometrics and Bioinformatics, Vol. 5: 4: 216- 224,2011

[23] P.P.S. Tomar, R. Singh \& P. K. Saxena, 2011, Multimedia Based MDSS For Chronic Lung Diseases Diagnosis Using Rule-based Technique, Afr J. of Comp \& ICTs, Vol 4, No. 3. Issue 2, pp. 1-6

[24] P.P.S. Tomar, Ranjit Singh, P K Saxena, B K Sharma, 2012, "A medical multimedia based DSS for heart diseases diagnosis and training," Canadian Journal on Biomedical Engineering \& Technology Vol. 3 ( 2), page 30-38

[25] Slade, S. ,1991, Case-based reasoning: A research paradigm. AI Magazine, 4(1), 42-55.

[26] Aamodt, A., \& Plaza, E., 1994, Case-based reasoning: Foundational issues, methodological variation and system approaches. Artificial Intelligence Communications, 7(1), 39-59.

[27] Turban, E., Aronson, J. E., Liang, T. \& Sharda, R., 2007, Decision Support and Business Intelligence Systems, Pearson Prentice Hall, Upper Saddle River.

[28] Sprague, R. H. and. Carlson, E. D ,1982, Building effective decision support systems. Englewood Cliffs, N.J., Prentice-Hall. ISBN 0-130-86215-0

[29] Sage, A. P. ,1991, Decision Support Systems Engineering. John Wiley \& Sons, Inc., New York. 


\section{AUTHOR'S PROFILE}

Prempal Singh Tomar has extensive and well-rounded experience of eleven years in Technical Education at UG and PG level. He received his B.E (Electronics Engineering) from Nagpur University, Nagpur, India and M.Tech in Electronics Design and Technology from Uttar Pradesh Technical University, Lucknow, India in 2003. He served as Assistant Professor of Electronics \& Communication at Hindustan College of Science \& Technology Mathura, India. He also served as Head of Department of Electronics \& Communication Engineering for four years at ACET, Aligarh and MIT, Bulandshahr, India. He is presently pursing $\mathrm{PhD}$ in Decision Support System at Faculty of Engineering, Dayalbagh Educational Institute, Agra, India. He was the member of accreditation team of Uttar Pradesh Technical University, Lucknow, India in year 2004 and 2005. He was the team member for the E-learning program for UG courses at Hindustan College of Science and Technology, Mathura, India. He designed and developed a SMPS to cancel EMI that followed the International standard for EMI (MIL-461 E). His research interests are Analog Integrated Circuits, Microwave Engineering, Software Engineering, Artificial Intelligence, and Decision Support Systems. He has published several research papers in international journals and conferences.

Ranjit Singh has received his B.Sc. Engineering in Mechanical Engineering from Agra University and his M.Tech. in Production Engineering from I.I.T. Roorkee and obtained a Gold Medal and done Ph.D. from Dayalbagh Educational Institute in Flexible Automation. Presently, he is
Professor in Mechanical Engineering at Dayalbagh Educational Institute, Dayalbagh, Agra. He has been involved actively in research and teaching since 1973. His research interests are intelligent manufacturing, foundry techno-logy, ergonomics, bio-medical engineering, and soft computing applications in manufacturing. He has guided, and continues to guide, Ph.D.'s in the above fields and has completed successfully three research projects granted by the Government of India. He has been awarded the prestigious Ramanna Fellowship for the year 2006 by the Dept. of Science and Technology, Government of India, and has received the most coveted $P$. Banerjee Medal for the best technical paper in the Indian Foundry Journal, 2000. Presently he is working on a research project sanctioned by UGC, New Delhi, India. He has 80 publications to his credit in journals and conferences. In addition, he has co-edited the proceedings of the National Systems Conference - 1994. He is a life member of the Institution of Engineers (India) and the Systems Society of India. He has chaired technical sessions at various conferences and workshops in India and Abroad.

P. K. Saxena received his doctoral degree in Industrial Engineering from I.I.T. Delhi, New Delhi, India; his research interests include Non- Linear programming, Information Systems, Decision Support Systems and Bio-Medical Engineering. His publications have appeared in various leading national and International journals. He retired as Dean, Faculty of Engineering, Dayalbagh Educational Institute, Agra, India and also served as Professor and Head, Department of Mechanical Engineering, Faculty of Engineering, Dayalbagh Educational Institute, Agra, India. 